\title{
EXTREMAL PATHS AND HOLONOMIC CONSTITUTIVE LAWS IN ELASTOPLASTICITY*
}

\author{
BY \\ B. D. REDDY ${ }^{1}$, J. B. MARTIN ${ }^{2}$, AND T. B. GRIFFIN ${ }^{3}$ \\ University of Cape Town, South Africa
}

\begin{abstract}
Extremal stress paths between any two stresses are investigated for elasticplastic materials, extending existing results which hold for the case when one of the stress points is at the origin. Assumptions about the differentiability of the various work and complementary work functions are relaxed, and it is shown that the maximum complementary work $\hat{U}$ is a potential for the strain in the sense that the strain lies in the subdifferential of $\hat{U}$. In the same way the minimum work $\hat{W}$ is a potential for stress. Parallel investigations with respect to maximum complementary plastic work and plastic work show that these quantities are potentials for stress and plastic strain increment, respectively. A holonomic constitutive law based on the relations between stress and strain, obtained when the stress history follows an extremal path, is constructed.
\end{abstract}

1. Introduction. The general problem of formulating constitutive laws for elastic-plastic materials is a complex one, given the path-dependent nature of the response of such materials. For example, consider the situation shown in Fig. 1.1, in which a value of stress $\sigma^{0}$ and corresponding strain $\varepsilon^{0}$ are given, and it is required to find the strain $\varepsilon$ corresponding to $\sigma$. A fundamental assumption about plastic material behavior is that $\varepsilon$ is not unique, but depends on the path traversed between $\sigma^{0}$ and $\sigma$. For this reason elastic-plastic constitutive laws are conventionally given in the rate form

$$
\dot{\boldsymbol{\varepsilon}}=\dot{\boldsymbol{\varepsilon}}\left(\dot{\boldsymbol{\sigma}}, \boldsymbol{\sigma}, h_{\alpha}\right), \quad()=\frac{d}{d t}(),
$$

where $h_{\alpha}$ is a set of internal variables and $t(0 \leqslant t \leqslant 1)$ parametrizes path length, say, so that $\varepsilon$ may be found from

$$
\boldsymbol{\varepsilon}=\varepsilon^{0}+\int_{0}^{1} \dot{\boldsymbol{\varepsilon}}\left(\dot{\mathbf{f}}(t), \mathbf{f}(t), h_{\alpha}(t)\right) d t
$$

for the path $\mathbf{f}(t)$ shown in Fig. 1.1.

\footnotetext{
* Received June 24, 1986.

${ }^{1}$ Department of Applied Mathematics

${ }^{2}$ Faculty of Engineering

${ }^{3}$ Department of Civil Engineering
} 


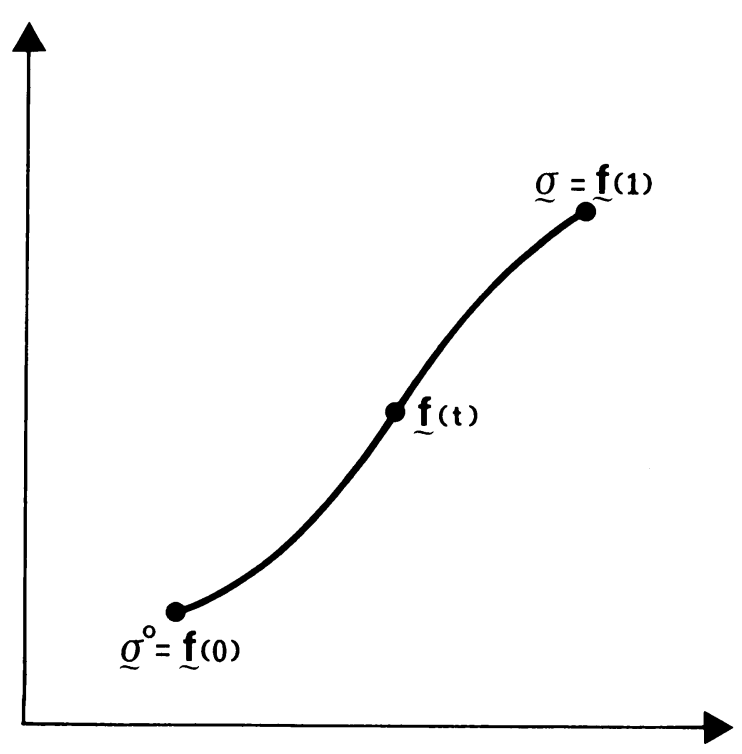

FIG. 1.1. A stress path between $\boldsymbol{\sigma}^{0}$ and $\boldsymbol{\sigma}$.

Various attempts have been made at formulating constitutive laws for elastic-plastic materials which give strain as a function of stress. Such theories, known as holonomic or deformation theories, are by their very nature approximations since they take no account of path-dependence. But if the range of stress paths to which they are applied is limited in some sense, then deformation theories provide reasonable approximations of the actual material behavior. Hencky (1924) and Nadai (1931) were among the first to propose deformation theories, but the widespread belief that such theories were limited strictly to proportional loading (and the fact that proportional loading was of limited practical significance) led to a certain amount of hestitation in their being accepted. Budiansky (1959) succeeded in dispelling this belief to some extent by showing that, using Nadai's theory, quite acceptable results could be obtained for loading paths which deviated considerably from proportional loading.

Within the context of mathematical programming approaches to elastic-plastic problems, Maier and his associates (Maier (1969), Cohn and Maier (1979)) have made extensive use of holonomic approaches; any load path which deviates considerably from radial is approximated by a piecewise linear path, and holonomic behavior is assumed within any linear section of the path.

In principle, any scheme or rule by means of which one associates with a stress point $\sigma$ a single path between a given stress point $\sigma^{0}$ and $\sigma$, is sufficient to establish a holonomic relation. For this we simply use (1.2) to establish the constitutive relation

$$
\varepsilon=\varepsilon^{0}+\mathbf{g}(\sigma) \equiv \tilde{\varepsilon}(\sigma) .
$$

This relation, being one-to-one, is invertible and we have

$$
\sigma=\tilde{\sigma}(\varepsilon)
$$


as the inverse relation. Once such a scheme for choosing paths has been decided upon and used to establish the relations (1.3) and (1.4), the next step is to define a fictitious nonlinear elastic material called a holonomic material, as one having the constitutive relations above. The use of these constitutive relations instead of the actual relations (1.1) will lead to results which differ in general from those corresponding to an elastic-plastic material, unless the stress paths arising in the problem do not differ radically from those used in the first place to set up (1.3). An alternative, converse approach is to postulate a constitutive relation of the form (1.3), and subsequently to investigate the range of stress paths for which it approximates reasonably the behavior of an elastic-plastic material. This is the case for the Hencky-Nadai theory, which is based on the assumption that the stress and strain deviators are proportional (see Budiansky (1959), Kachanov (1974)). It can be shown that the theory is valid for a range of stress paths other than radial paths. The theory is established for stress paths emanating from the origin, i.e., $\boldsymbol{\sigma}^{0}=\mathbf{0}$.

The first approach, namely that which starts by establishing a rule for choosing stress paths, has been used by Martin, Ponter, and others (see Ponter and Martin (1972), and Martin (1975) for a review), who associate with each stress point $\sigma$ a path emanating from the origin which has the property that the complementary work $\hat{U}$ along this path is a maximum (Fig. 1.2). This choice of path has far-reaching, and very advantageous consequences. It can be shown that the work function $\hat{W}$ along the strain path corresponding to the extremal stress path is a minimum, and furthermore that $\hat{U}$ and $\hat{W}$ are, respectively, potential functions for strain $\varepsilon$ and stress $\sigma$ :

$$
\varepsilon=\partial \hat{U} / \partial \boldsymbol{\sigma}, \quad \boldsymbol{\sigma}=\partial \hat{W} / \partial \boldsymbol{\varepsilon} .
$$

A holonomic material with the constitutive relations (1.5) makes for a well-posed variational theory for the associated boundary-value problem, given that $\hat{U}$ and $\hat{W}$ are shown also to be convex.

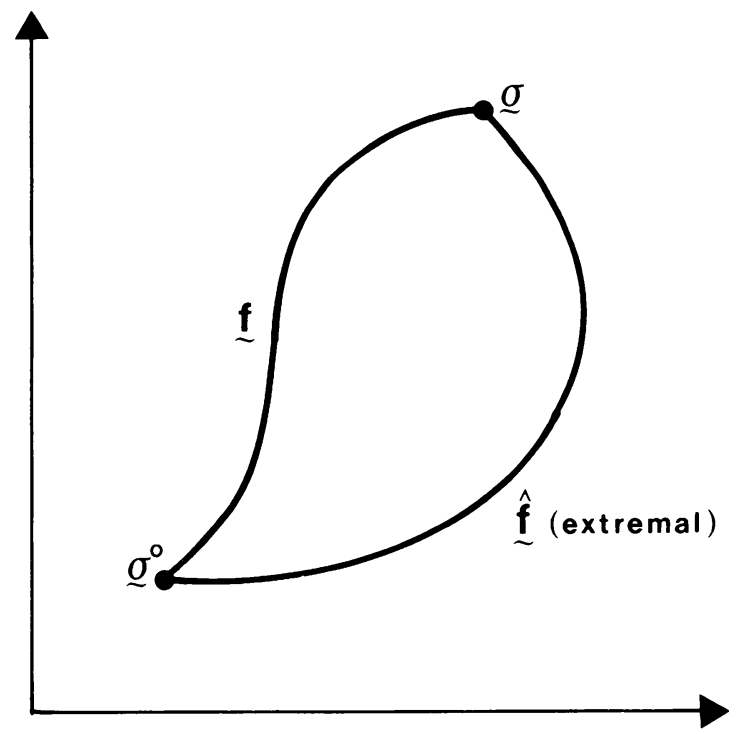

Fig. 1.2. An extremal path in stress space. 
This procedure may likewise be applied to the complementary plastic work function $U^{p}$ and the plastic work function $W^{p}$ (Ponter and Martin (1972)); results similar to (1.5) are found, with $\varepsilon, \hat{U}$, and $\hat{W}$ replaced by plastic strain $\mathbf{p}$, the maximum complementary plastic work $\hat{U}^{p}$ and minimum plastic work $\hat{W}^{p}$, respectively.

The aim of the present contribution is to extend and place on a firmer foundation the Martin-Ponter theory, in a variety of ways. First, we consider extremal stress paths emanating from a fixed but arbitrary nonzero stress point $\boldsymbol{\sigma}^{0}$ with corresponding strain $\boldsymbol{\varepsilon}^{0}$, and terminating at a point $\boldsymbol{\sigma}$. Secondly, no assumptions of differentiability are made with respect to the work and complementary work functions; it is still possible to show that these functions are convex, and (1.5) are replaced by statements to the effect that $\varepsilon$ and $\boldsymbol{\sigma}$ lie, respectively, in the subdifferentials of $\hat{U}$ and $\hat{W}$ :

$$
\varepsilon \in \partial \hat{U}(\boldsymbol{\sigma}), \quad \boldsymbol{\sigma} \in \partial \hat{W}(\varepsilon) .
$$

Similar results are obtained with respect to plastic work and complementary plastic work. We also introduce the idea of treating the complementary work and work functions as mappings of a path to a scalar: for example, as we see in Sec. 2, $U$ is defined by

$$
U: H \rightarrow R, \quad U(\mathbf{f})=\int_{0}^{1} \tilde{\varepsilon}(\mathbf{f}(t)) \cdot \dot{\mathbf{f}}(t) d t,
$$

where $H$ is an appropriately defined space of functions and $\mathbf{f}(t)$ is the path between $\boldsymbol{\sigma}^{0}$

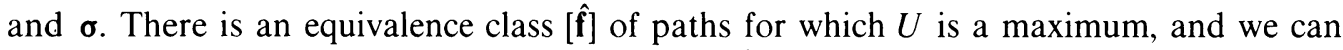

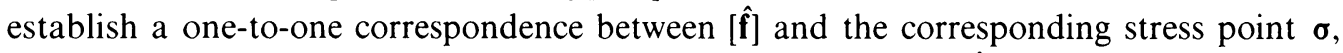
from which follows the maximum complementary work function $\hat{U}$ :

$$
\hat{U}: R^{N \times N} \rightarrow R, \quad \hat{U}(\boldsymbol{\sigma})=U(\hat{\mathbf{f}}) \text { for } \hat{\mathbf{f}} \in[\hat{\mathbf{f}}] .
$$

Similar considerations apply with respect to the work function.

We rework Martin and Ponter's treatment of extremal complementary plastic work $U^{p}$ and plastic work $W^{p}$ in this more general framework. In particular, we avoid the use of the multivalued function $\boldsymbol{\sigma}(\mathbf{p})$, where $\mathbf{p}$ is plastic strain, in the definition of plastic work $W^{p}$. The plastic work is instead defined by making use of complementary plastic work together with conjugacy arguments.

We show that the extremal functions $\hat{U}^{p}$ and $\hat{W}^{p}$ are convex (a result missing from the original treatment) and that $\hat{U}^{p}$ and $\hat{W}^{p}$ are, respectively, potentials for plastic strain and stress.

The fact that we develop a holonomic theory for what are, in effect, initially stressed materials allows us to implement the theory proposed here in an incremental fashion in a boundary-value problem. Suppose, for example, that it is required to solve an elastic-plastic problem for a load history $\mathbf{P}(t)$ such as that shown in Fig. 1.3. A single-step holonomic theory which is valid for straight-line paths only, say, will generally produce unacceptable answers, but if we divide $\mathbf{P}(t)$ into segments as shown in the figure and solve first for the response due to the load $\mathbf{P}\left(t_{1}\right)$ using a holonomic theory, and next for the response at $\mathbf{P}\left(t_{2}\right)$ using the state at $\mathbf{P}\left(t_{1}\right)$ as an initial state, and so on, then a more acceptable result is to be expected. This aspect is treated in greater detail in a separate contribution (see Griffin, Reddy, and Martin (1986)). 


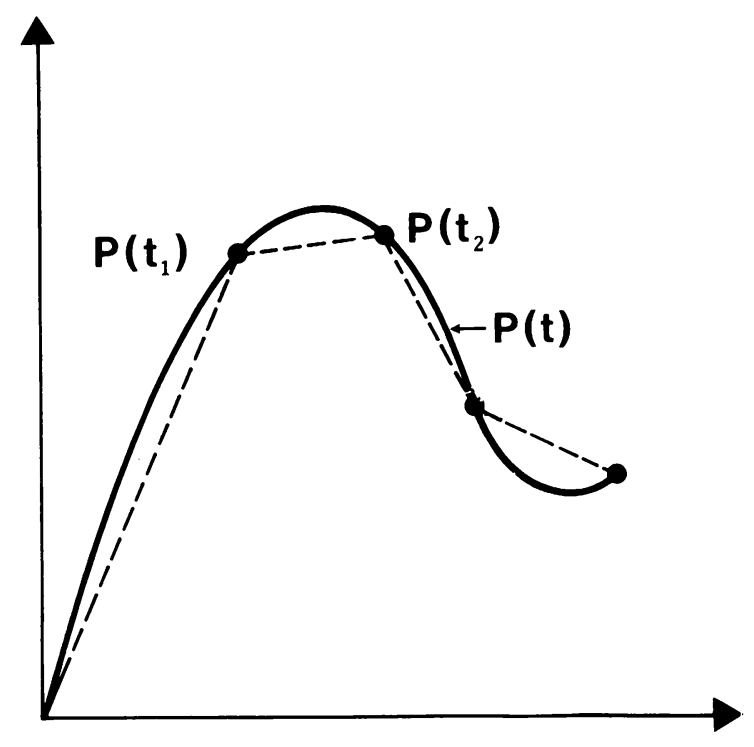

FIG. 1.3. A loading history $\mathbf{P}(t)$.

2. Extremal stress paths and complementary work. Consider a material point which has associated with it a stress $\boldsymbol{\sigma}^{0}$ and corresponding strain $\varepsilon^{0}$. Suppose that the stress $\boldsymbol{\sigma}^{0}$ is changed to a value $\boldsymbol{\sigma}$ along a path $\mathbf{f}(t)(0 \leqslant t \leqslant 1)$, where $\mathbf{f}(0)=\boldsymbol{\sigma}^{0}$ and $\mathbf{f}(1)=\boldsymbol{\sigma}$ (Fig. 1.1). Corresponding to this change in stress there will be a change in strain, from $\varepsilon^{0}$ to a value $\varepsilon$. The strain $\varepsilon$ is related to the stress $\sigma$ via an appropriate constitutive relation, and if this constitutive relation is path-dependent, then the strain $\varepsilon$ will depend not only on $\sigma$ but also on the path $\mathbf{f}(t)$ between $\boldsymbol{\sigma}^{0}$ and $\boldsymbol{\sigma}$.

We assume that the material point is part of an elastic-plastic material, which at this stage it suffices to characterize by requiring that the three following postulates are obeyed.

Postulate 1 . The strain $\varepsilon$ corresponding to a stress $\sigma$ may be decomposed into an elastic component $\mathbf{e}$ and a plastic component $\mathbf{p}: \varepsilon=\mathbf{e}+\mathbf{p}$. The elastic strain $\mathbf{e}$ is uniquely determined by $\boldsymbol{\sigma}$, and is given by

$$
\mathbf{e}=\mathbf{D} \boldsymbol{\sigma},
$$

where $\mathbf{D}$ is a fourth-order tensor of elastic moduli, while the plastic strain $\mathbf{p}$ is a function of $\boldsymbol{\sigma}$ as well as of the path from $\boldsymbol{\sigma}^{0}$ to $\boldsymbol{\sigma}$. Corresponding to any given stress path $\mathbf{f}(t)$ with $\mathbf{f}(0)=\boldsymbol{\sigma}^{0}$, the plastic strain $\mathbf{p}(t)$ is a single-valued function of stress:

$$
\mathbf{p}=\tilde{\mathbf{p}}(\boldsymbol{\sigma}) \text { or } \mathbf{p}(t)=\tilde{\mathbf{p}}(\mathbf{f}(t)), \quad \mathbf{p}(0)=\mathbf{p}^{0} .
$$

Thus the strain is also a single-valued function of stress:

$$
\varepsilon=\mathbf{D} \boldsymbol{\sigma}+\tilde{\mathbf{p}}(\boldsymbol{\sigma}) \equiv \tilde{\boldsymbol{\varepsilon}}(\boldsymbol{\sigma})
$$

or

$$
\varepsilon(t)=\tilde{\varepsilon}(\mathbf{f}(t)), \quad \varepsilon(0)=\varepsilon^{0} .
$$


Postulate 2. Let $\boldsymbol{\sigma}^{1}$ and $\boldsymbol{\sigma}^{2}$ be arbitrary values of stress, with corresponding values of strain $\varepsilon^{1}=\mathbf{e}^{1}+\mathbf{p}^{1}$ and $\varepsilon^{2}=\mathbf{e}^{2}+\mathbf{p}^{2}$. If $\boldsymbol{\sigma}^{2}$ is reached from $\sigma^{1}$ along a straight-line path $\mathbf{f}(t)=(1-t) \boldsymbol{\sigma}^{1}+t \boldsymbol{\sigma}^{2}, 0 \leqslant t \leqslant 1$, then

$$
\left(\boldsymbol{\sigma}^{1}-\boldsymbol{\sigma}^{2}\right) \cdot\left(\mathbf{p}^{1}-\mathbf{p}^{2}\right) \geqslant 0 .
$$

Postulate 3. Let $\mathbf{f}(t)(0 \leqslant t \leqslant 1)$ be any closed stress path, that is, $\mathbf{f}(0)=\mathbf{f}(1)$. Then

$$
\oint \varepsilon \cdot d \boldsymbol{\sigma} \leqslant 0 \text { or } \int_{0}^{1} \tilde{\varepsilon}(\mathbf{f}(t)) \cdot \dot{\mathbf{f}}(t) d t \leqslant 0, \quad \mathbf{f}(0)=\mathbf{f}(1) .
$$

In addition to the above postulates we make the usual assumption that the elasticity tensor $\mathbf{D}$ is strongly elliptic:

$$
\boldsymbol{\sigma} \cdot \mathbf{D} \boldsymbol{\sigma} \equiv D_{i j k l} \boldsymbol{\sigma}_{i j} \sigma_{k l} \geqslant \mu|\boldsymbol{\sigma}|^{2} \text { for some } \mu>0 .
$$

A comment on our use of the above postulates is in order. Postulates 1 to 3 are sufficient for the construction of the classical theory of plasticity, but these postulates are often found in slightly different, though equivalent forms. Martin (1975), for example, uses instead of $(2.4)$ the inequality $\left(\sigma^{1}-\sigma^{2}\right) \cdot\left(\varepsilon^{1}-\varepsilon^{2}\right) \geqslant 0$, which can be reached as a consequence of (2.1), (2.4), and (2.6). In what follows, we will assume at all times that postulates 1 to 3 hold.

Suppose, then, that we are given a material point with values $\sigma^{0}$ and $\varepsilon^{0}$ of stress and strain. Along any given stress path $\mathbf{f}(t)(0 \leqslant t \leqslant 1)$ emanating from $\boldsymbol{\sigma}^{0}$ and terminating at $\boldsymbol{\sigma}$ the strain is determined uniquely by $\varepsilon(t)=\tilde{\varepsilon}(\mathbf{f}(t))$. Here $\mathbf{f}(0)=\boldsymbol{\sigma}^{0}$ and $\mathbf{f}(1)=\boldsymbol{\sigma}$. We define the complementary work $U$ associated with such a path by

$$
U: H_{\sigma} \rightarrow R, U(\mathbf{f})=\int_{0}^{1} \tilde{\varepsilon}(\mathbf{f}(t)) \cdot \dot{\mathbf{f}}(t) d t,
$$

where $H_{\sigma}=\left\{\mathbf{f}: \mathbf{f}=\left(f_{i j}\right), \quad f_{i j} \in H^{1}(0,1), \mathbf{f}(0)=\boldsymbol{\sigma}^{0}, \mathbf{f}(1)=\boldsymbol{\sigma}\right\} \quad$ and $\quad H^{1}(0,1)=\{v \in$ $\left.L_{2}(0,1): \dot{v} \in L_{2}(0,1)\right\}$, where $L_{2}(0,1)$ is the space of (Lebesgue-) square-integrable functions on $(0,1)$.

An extremal path $\hat{\mathbf{f}} \in H_{\sigma}$ is defined to be one for which

$$
U(\hat{\mathbf{f}}) \geqslant U(\mathbf{f}) \text { for all } \mathbf{f} \in H_{\sigma} .
$$

We introduce next

Lemma 2.1. The Complementary Work Inequality. Let $\hat{\mathbf{f}}(t)(0 \leqslant t \leqslant 1)$ be an extremal path between $\boldsymbol{\sigma}^{0}$ and $\boldsymbol{\sigma}$ with complementary work $U(\hat{\mathbf{f}})$, and let $\mathbf{g}(t)(0 \leqslant t \leqslant 1)$ be an arbitrary path between $\sigma^{0}$ and $\sigma^{*}(\neq \sigma)$, with complementary work $U(\mathbf{g})$. If $\tilde{\varepsilon}\left(\sigma^{*}\right)$ is the strain corresponding to $\sigma^{*}$ then

$$
U(\hat{\mathbf{f}})-U(\mathbf{g})-\tilde{\varepsilon}\left(\boldsymbol{\sigma}^{*}\right) \cdot\left(\boldsymbol{\sigma}-\boldsymbol{\sigma}^{*}\right) \geqslant 0 .
$$

Proof. The proof follows without modification that given by Ponter and Martin (1972) (see also Martin (1975), page 726) for the case $\boldsymbol{\sigma}^{0}=\mathbf{0}$.

Extremal stress paths are, in general, not unique. To take one example, suppose that $\boldsymbol{\sigma}^{0}$ lies in the elastic region, that is, in the region bounded by the initial yield surface $\phi(\boldsymbol{\sigma})=0$, and $\boldsymbol{\sigma}$ lies outside this region. Then if $\hat{\mathbf{f}}$ is an extremal path from $\boldsymbol{\sigma}^{0}$ to $\boldsymbol{\sigma}$, so is 
any other path $\hat{\mathbf{g}}$ which differs from $\hat{\mathbf{f}}$ only in the elastic region (Fig. 2.1), since the complementary work between two points in the elastic region is path-independent.

We can, however, define an equivalence class $[\hat{\mathbf{f}}]$ of extremal paths between $\boldsymbol{\sigma}^{0}$ and $\boldsymbol{\sigma}$ by

$$
[\hat{\mathbf{f}}]_{\sigma}=\left\{\mathbf{g} \in H_{\sigma}: U(\mathbf{g})=U(\hat{\mathbf{f}})\right\}
$$

then if we define the set of equivalence classes of extremal paths between $\sigma^{0}$ and all points $\boldsymbol{\sigma}$ in $R^{N \times N}$ by

$$
\hat{H}_{\sigma}=\left\{[\hat{\mathbf{f}}]_{\sigma} \subset H_{\sigma}: \sigma \in R^{N \times N}\right\},
$$

we observe that there is a one-to-one correspondence between elements of $\hat{H}_{\sigma}$ and stress points in $R^{N \times N}$. We denote this correspondence by

$$
A: \hat{H}_{\sigma} \rightarrow R^{N \times N}, \quad A(\hat{\mathbf{f}})=\sigma \text { for any } \hat{\mathbf{f}} \in[\hat{\mathbf{f}}] .
$$

It follows that we may define the function $\hat{U}$ by

$$
\hat{U}: R^{N \times N} \rightarrow R, \quad \hat{U}=U \circ A^{-1}, \quad \text { or } \hat{U}(\boldsymbol{\sigma})=U\left(A^{-1}(\boldsymbol{\sigma})\right)=U(\hat{\mathbf{f}}) .
$$

From Lemma 2.1 follows

THEOREM 2.1. (a) The complementary work functional $\hat{U}: R^{N \times N} \rightarrow R$ is convex.

(b) The strain $\varepsilon$ corresponding to a stress $\sigma$ reached along an extremal stress path belongs to the subdifferential of $\hat{U}$, that is,

$$
\varepsilon=\tilde{\varepsilon}(\boldsymbol{\sigma}) \in \partial \hat{U}(\boldsymbol{\sigma})=\{\boldsymbol{\eta}: \hat{U}(\tau)-\hat{U}(\boldsymbol{\sigma})-\boldsymbol{\eta} \cdot(\tau-\boldsymbol{\sigma}) \geqslant 0\},
$$

so that

$$
\tilde{\boldsymbol{\varepsilon}}(\boldsymbol{\sigma})=\left.\frac{\partial \hat{U}}{\partial \boldsymbol{\sigma}}\right|_{\sigma},
$$

if $\hat{U}$ is differentiable at $\boldsymbol{\sigma}$.

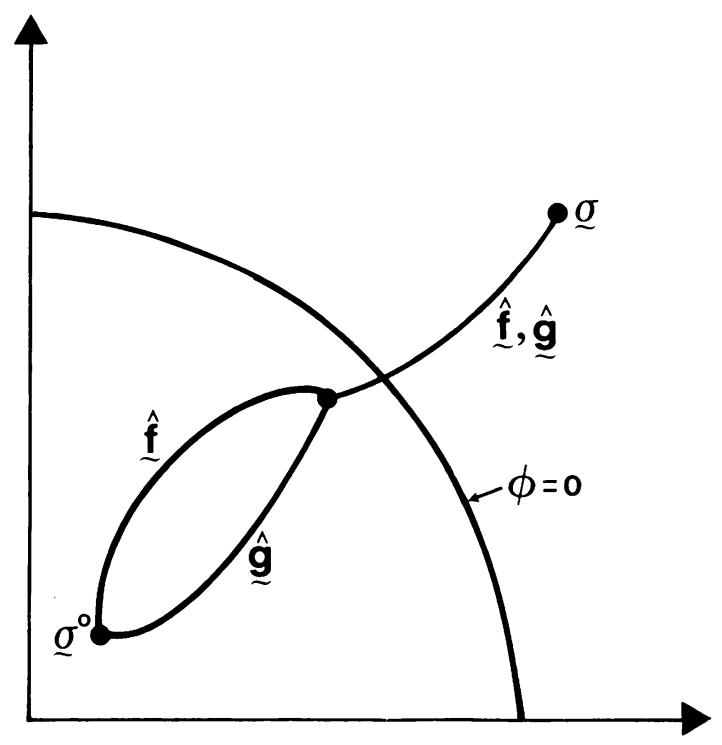

FIG. 2.1. Two extremal paths between $\boldsymbol{\sigma}^{0}$ and $\boldsymbol{\sigma}$. 
Proof. (a) The convexity of $\hat{U}$ follows from (2.9). Choose $\mathbf{g}$ in Lemma 2.1 to be an extremal path from $\sigma^{0}$ to $\sigma^{*}$. Then setting $\sigma^{*}=\theta \sigma+(1-\theta) \tau$ in $(2.9)(0<\theta<1)$ and multiplying throughout by $\theta$, we have

$$
\theta \hat{U}(\boldsymbol{\sigma})-\theta \hat{U}(\theta \boldsymbol{\sigma}+(1-\theta) \tau)-\theta(1-\theta) \tilde{\varepsilon}(\theta \boldsymbol{\sigma}+(1-\theta) \tau) \cdot(\sigma-\tau) \geqslant 0 .
$$

Next, setting $\sigma=\tau, \sigma^{*}=\theta \sigma+(1-\theta) \tau$, and multiplying throughout by $(1-\theta)$, we obtain

$$
(1-\theta) \hat{U}(\tau)-(1-\theta) \hat{U}(\theta \sigma+(1-\theta) \tau)+\theta(1-\theta) \tilde{\varepsilon}(\theta \sigma+(1-\theta) \tau) \cdot(\sigma-\tau) \geqslant 0
$$

Adding, we get $\hat{U}(\theta \sigma+(1-\theta) \tau) \leqslant \theta \hat{U}(\sigma)+(1-\theta) \hat{U}(\tau)$, which confirms the convexity of $\hat{U}$.

(b) That $\varepsilon(\boldsymbol{\sigma})$ lies in the subdifferential of $\hat{U}$ at $\boldsymbol{\sigma}$ follows from (2.9) by making $\mathbf{g}$ an extremal path. Furthermore, from the convexity of $\hat{U}$ it follows from a standard result in convex analysis (Aubin and Ekeland (1984), Ekeland and Temam (1976), Chapter 1, Proposition 5.3, Rockafeller (1970), Part V) that if $\hat{U}$ is differentiable at $\sigma$, then $\partial \hat{U}(\boldsymbol{\sigma})=\{\partial \hat{U} / \partial \boldsymbol{\sigma}\}$. This concludes the proof.

The proof of Theorem 2.1 differs from that given by Ponter and Martin (1972), who assume the differentiability of $\hat{U}$ at the outset. Of course, differentiability of $\hat{U}$ is a necessary property if we are to expect $\varepsilon(\boldsymbol{\sigma})$ corresponding to a given path to be single-valued. When $\hat{U}$ is differentiable it is a potential function for the strain, and we are then at liberty to define a fictitious nonlinear elastic material with constitutive relation $\boldsymbol{\varepsilon}=\partial \hat{U} / \partial \boldsymbol{\sigma}$, the elastic behavior being inferred from the property of path-independence:

$$
\oint \hat{\boldsymbol{\varepsilon}} \cdot d \boldsymbol{\sigma}=0 \text {, }
$$

where $\hat{\boldsymbol{\varepsilon}}=\partial \hat{U} / \partial \boldsymbol{\sigma}$. Such a material coincides with an elastic-plastic material only in the event that the stress path followed is an extremal path. We call this fictitious material a holonomic material.

3. Extremal strain paths and work. An analogous development may be carried out with respect to paths in strain space. Consider a material point with stress $\sigma^{0}$ and strain $\varepsilon^{0}$, and let $\mathbf{f}(t), 0 \leqslant t \leqslant 1$, be a stress path from $\boldsymbol{\sigma}^{0}$ to $\boldsymbol{\sigma}$. The map $\tilde{\varepsilon}$ defined in (2.3) allows us to define a corresponding strain path $\mathbf{b}(t), 0 \leqslant t \leqslant 1$, by $\mathbf{b}=\tilde{\varepsilon} \circ \mathbf{f}$ or $\mathbf{b}(t)=\tilde{\varepsilon}(\mathbf{f}(t))$, with

$$
\mathbf{b}(0)=\varepsilon^{0}, \quad \mathbf{b}(1)=\varepsilon=\tilde{\varepsilon}(\boldsymbol{\sigma}) .
$$

The work $W$ associated with the strain path $\mathbf{b}$ is defined by

$$
W: H_{\varepsilon} \rightarrow R, W(\mathbf{b})=\int_{0}^{1} \tilde{\boldsymbol{\sigma}}(\mathbf{b}(t)) \cdot \dot{\mathbf{b}}(t) d t,
$$

where $\tilde{\boldsymbol{\sigma}}=\tilde{\boldsymbol{\varepsilon}}^{-1}$ and

$$
H_{\varepsilon}=\left\{\mathbf{b}: \mathbf{b}=\left(b_{i j}\right), b_{i j} \in H^{1}(0,1), \mathbf{b}(0)=\varepsilon^{0}, \mathbf{b}(1)=\varepsilon\right\} .
$$

An extremal strain path $\hat{\mathbf{b}} \in H_{\mathrm{r}}$ is defined to be one for which

$$
W(\hat{\mathbf{b}}) \leqslant W(\mathbf{b}) \text { for all } \mathbf{b} \in H_{\varepsilon} .
$$

As in the case of extremal stress paths, there is a one-to-one correspondence between the set $\hat{H}_{\varepsilon}$ of equivalence classes of extremal strain paths and the set of strains, which is denoted by

$$
B: \hat{H}_{\varepsilon} \rightarrow R^{N \times N}, \quad B(\mathbf{b})=\varepsilon \text { for } \mathbf{b} \in[\hat{\mathbf{b}}] .
$$


We define the function $\hat{W}$ by

$$
\hat{W}: R^{N \times N} \rightarrow R, \quad \hat{W}=W \circ B^{-1}, \quad \text { or } \hat{W}(\varepsilon)=W\left(B^{-1}(\varepsilon)\right)=W(\hat{\mathbf{b}}) .
$$

We now record a few properties of extremal strain paths and the extremal work function $\hat{W}$.

THEOREM 3.1. (a) If $\hat{\mathbf{f}}(t)(0 \leqslant t \leqslant 1)$ is an extremal stress path between $\boldsymbol{\sigma}^{0}$ and $\boldsymbol{\sigma}$ then $\hat{\mathbf{b}}(t) \equiv \tilde{\mathbf{\varepsilon}}(\hat{\mathbf{f}}(t))$ is an extremal strain path.

(b) The work function $\hat{W}$ defined by (3.4) is convex.

(c) $\sigma=\tilde{\sigma}(\varepsilon) \in \partial \hat{W}(\varepsilon)$, so that

$$
\tilde{\boldsymbol{\sigma}}(\boldsymbol{\varepsilon})=\left.\frac{\partial \hat{W}}{\partial \boldsymbol{\varepsilon}}\right|_{\boldsymbol{\varepsilon}}
$$

if $\hat{W}$ is differentiable at $\varepsilon$.

Proof. (a) Let $\hat{\mathbf{f}}$ and $\mathbf{f}$ be two stress paths satisfying $\hat{\mathbf{f}}(0)=\mathbf{f}(0)=\boldsymbol{\sigma}^{0}, \hat{\mathbf{f}}(1)=\boldsymbol{\sigma}^{1}$ and $\mathbf{f}(1)=\boldsymbol{\sigma}^{2}$, and set $\tilde{\boldsymbol{\varepsilon}}(\hat{\mathbf{f}}(t))=\hat{\mathbf{b}}(t), \boldsymbol{\varepsilon}(\mathbf{f}(t))=\mathbf{b}(t)$, with $\hat{\mathbf{b}}(1)=\mathbf{b}(1)=\varepsilon^{*}$. In other words, the two stress paths $\hat{\mathbf{f}}$ and $\mathbf{f}$ are mapped into strain paths which commence and terminate at the same points (Fig. 3.1). We have

$$
\int_{0}^{1} \tilde{\boldsymbol{\varepsilon}}(\hat{\mathbf{f}}(t)) \cdot \dot{\hat{\mathbf{f}}}(t) d t+\int_{0}^{1} \tilde{\boldsymbol{\sigma}}(\hat{\mathbf{b}}(t)) \cdot \dot{\hat{\mathbf{b}}}(t) d t=\boldsymbol{\sigma}^{1} \cdot \boldsymbol{\varepsilon}^{*}-\boldsymbol{\sigma}^{0} \cdot \varepsilon^{0},
$$

or

$$
U(\hat{\mathbf{f}})+W(\hat{\mathbf{b}})=\boldsymbol{\sigma}^{1} \cdot \varepsilon^{*}-\boldsymbol{\sigma}^{0} \cdot \boldsymbol{\varepsilon}^{0},
$$

and

$$
\int_{0}^{1} \tilde{\varepsilon}(\mathbf{f}(t)) \cdot \dot{\mathbf{f}}(t) d t+\int_{0}^{1} \tilde{\boldsymbol{\sigma}}(\mathbf{b}(t)) \cdot \dot{\mathbf{b}}(t) d t=\boldsymbol{\sigma}^{2} \cdot \boldsymbol{\varepsilon}^{*}-\boldsymbol{\sigma}^{0} \cdot \varepsilon^{0}
$$

or

$$
U(\mathbf{f})+W(\mathbf{b})=\boldsymbol{\sigma}^{2} \cdot \varepsilon^{*}-\mathbf{\sigma}^{0} \cdot \varepsilon^{0} .
$$

From Lemma $2.1, U(\hat{\mathbf{f}})-\boldsymbol{\sigma}^{1} \cdot \varepsilon^{*} \geqslant U(\mathbf{f})-\boldsymbol{\sigma}^{2} \cdot \varepsilon^{*}$, whence $W(\hat{\mathbf{b}}) \leqslant W(\mathbf{b})$; in other words, the extremal stress path $\hat{\mathbf{f}}$ is mapped to the strain path $\hat{\mathbf{b}}$ which is an extremal strain path, so in effect we may write $W(\hat{\mathbf{b}})=\hat{W}\left(\varepsilon^{*}\right)$.
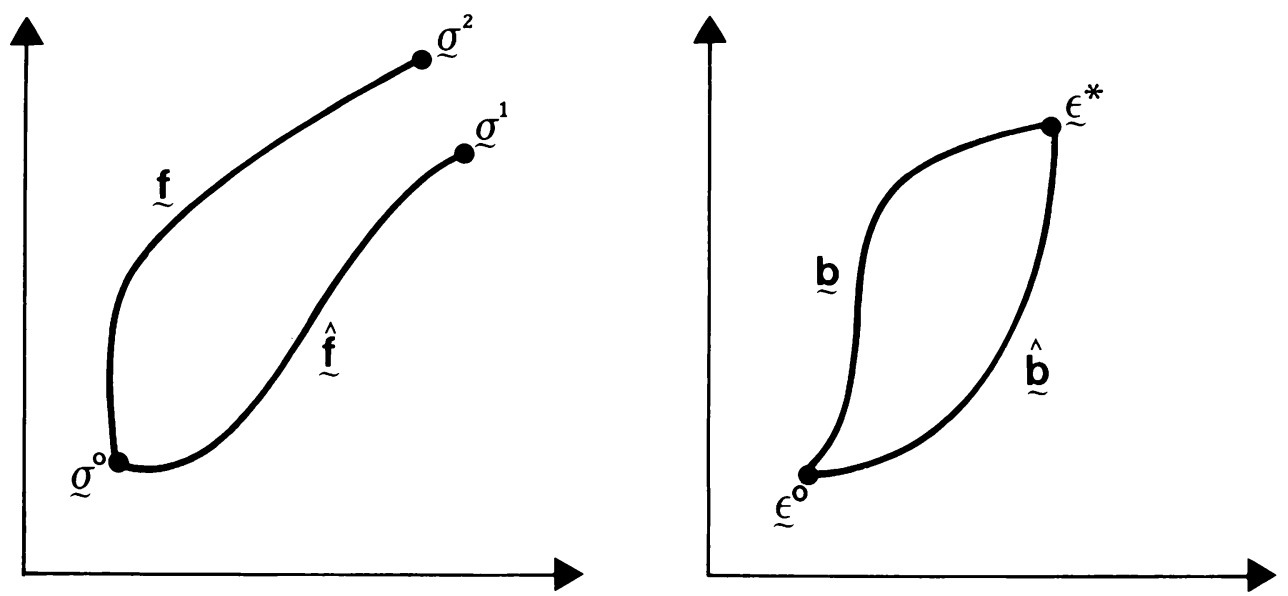

FIG. 3.1. Strain paths b, $\mathbf{b}$ corresponding to stress paths $\mathbf{f}, \hat{\mathbf{f}}$. 
(b) Let $\hat{\mathbf{f}}$ and $\hat{\mathbf{f}}$ be two extremal paths emanating from $\boldsymbol{\sigma}^{0}$ and terminating at $\boldsymbol{\sigma}^{1}, \boldsymbol{\sigma}^{2}$, with images the extremal strain paths $\hat{\mathbf{b}}$ and $\hat{\mathbf{b}}$ emanating from $\varepsilon^{0}$ and terminating at $\varepsilon^{1}$ and $\varepsilon^{2}$. Then

$$
\hat{U}\left(\boldsymbol{\sigma}^{i}\right)+\hat{W}\left(\boldsymbol{\varepsilon}^{i}\right)=\boldsymbol{\sigma}^{i} \cdot \boldsymbol{\varepsilon}^{i}-\boldsymbol{\sigma}^{0} \cdot \boldsymbol{\varepsilon}^{0}, \quad i=1,2 .
$$

The use of Lemma 2.1 leads to

$$
\hat{W}\left(\varepsilon^{1}\right)-\hat{W}\left(\varepsilon^{2}\right)-\sigma^{2} \cdot\left(\varepsilon^{1}-\varepsilon^{2}\right) \geqslant 0 .
$$

An argument similar to that used in the proof of Theorem 2.1(a) then gives the required result.

(c) The proof of (c) follows that of Theorem 2.1(b).

4. Complementary plastic work and plastic work. The additive decomposition of the strain into elastic and plastic components allows the possibility of defining work and complementary work functionals associated with the elastic and plastic strains. Let $\mathbf{f}(t)$ be a stress path with $\mathbf{f}(0)=\boldsymbol{\sigma}^{0}$ and $\mathbf{f}(1)=\boldsymbol{\sigma}$; then along this path the strain $\varepsilon$ and elastic strain e are found from

$$
\boldsymbol{\varepsilon}=\tilde{\varepsilon}(\boldsymbol{\sigma}), \quad \mathbf{e}=\mathbf{D} \boldsymbol{\sigma},
$$

so that

$$
\tilde{\mathbf{p}}(\boldsymbol{\sigma})=\tilde{\boldsymbol{\varepsilon}}(\boldsymbol{\sigma})-\mathbf{D} \boldsymbol{\sigma}
$$

Thus,

$$
U(\mathbf{f})=\underbrace{\int_{0}^{1}\left[\mathbf{D f}(t)+\mathbf{p}^{0}\right] \cdot \dot{\mathbf{f}}(t) d t}_{U^{\mathrm{comp}}(\mathbf{f})}+\underbrace{\int_{0}^{1} \Delta \tilde{\mathbf{p}}(\mathbf{f}(t)) \cdot \dot{\mathbf{f}}(t) d t}_{U^{p}(\mathbf{f})},
$$

where $\mathbf{p}^{0}$ is the plastic strain at $\boldsymbol{\sigma}^{0}$ and $\Delta \mathbf{p}=\mathbf{p}-\mathbf{p}^{0}$, so that $\Delta \tilde{\mathbf{p}}(\boldsymbol{\sigma})=\tilde{\mathbf{p}}(\boldsymbol{\sigma})-\mathbf{p}^{0}, U^{\text {comp }}$ is the complementary energy function between $\boldsymbol{\sigma}^{0}$ and $\boldsymbol{\sigma}$, while $U^{p}$ is called the complementary plastic work function. The integral defining $U^{\text {comp }}$ is path-independent so that $U$ derives its path-dependence from $U^{p}$.

In view of the path-independence of $U^{\text {comp }}$ it makes sense to define the function $U^{e}$ by

$$
U^{e}: R^{N \times N} \rightarrow R, \quad U^{e}(\boldsymbol{\sigma})=U^{\mathrm{comp}}(\mathbf{f})
$$

for any path $\mathbf{f}(t)$ between $\boldsymbol{\sigma}^{0}$ and $\boldsymbol{\sigma}$; indeed, we have

$$
U^{e}(\boldsymbol{\sigma})=\frac{1}{2}\left(\boldsymbol{\sigma}-\boldsymbol{\sigma}^{0}\right) \cdot \mathbf{D}\left(\boldsymbol{\sigma}-\boldsymbol{\sigma}^{0}\right)+\boldsymbol{\varepsilon}^{0} \cdot\left(\boldsymbol{\sigma}-\boldsymbol{\sigma}^{0}\right) .
$$

It follows also that if $\hat{\mathbf{f}}$ is an extremal stress path for $U$, then $\hat{\mathbf{f}}$ is at the same time an extremal path for $U^{p}$, since

$$
\begin{aligned}
U^{p}(\hat{\mathbf{f}}) & =U(\hat{\mathbf{f}})-U^{\mathrm{comp}}(\hat{\mathbf{f}}) \\
& \geqslant U(\mathbf{f})-U^{\operatorname{comp}}(\hat{\mathbf{f}})=U(\mathbf{f})-U^{\mathrm{comp}}(\mathbf{f}) \\
& =U^{p}(\mathbf{f}), \quad \mathbf{f} \in H_{\sigma} .
\end{aligned}
$$

As before we establish a one-to-one correspondence between members of $\hat{H}_{\sigma}$ and stress points, so that we may define by analogy with $(2.10)$ the function $\hat{U}^{p}: R^{N \times N} \rightarrow R$ by

$$
\hat{U}^{p}(\boldsymbol{\sigma})=U^{p}(\hat{\mathbf{f}}) \text {. }
$$

By using the second postulate, (2.4), it is possible to derive a complementary plastic work inecuality in much the same way as (2.9) is derived. We summarize this result in 
Lemma 4.1. Let $\hat{\mathbf{f}}(t)(0 \leqslant t \leqslant 1)$ be an extremal stress path with $\hat{\mathbf{f}}(0)=\boldsymbol{\sigma}^{0}, \hat{\mathbf{f}}(1)=\boldsymbol{\sigma}$, and let $\mathbf{g}(t)(0 \leqslant t \leqslant 1)$ be a stress path, not necessarily extremal, such that $\mathbf{g}(0)=\boldsymbol{\sigma}^{0}$ and $\mathbf{g}(1)=\sigma^{*} \neq \sigma$. Then,

$$
U^{p}(\hat{\mathbf{f}})-U^{p}(\mathbf{g})-\Delta \tilde{\mathbf{p}}\left(\boldsymbol{\sigma}^{*}\right) \cdot\left(\boldsymbol{\sigma}-\boldsymbol{\sigma}^{*}\right) \geqslant 0 .
$$

From Lemma 4.1 we immediately deduce results parallel to those embodied in Theorem 2.1. We summarize these results below.

THEOREM 4.1. (a) The function $\hat{U}^{p}: R^{N \times N} \rightarrow R$ is convex.

(b) $\Delta \mathbf{p} \in \partial \hat{U}^{p}(\boldsymbol{\sigma})$. Thus $\Delta \tilde{\mathbf{p}}(\boldsymbol{\sigma})=\partial \hat{U}^{p} / \partial \boldsymbol{\sigma}$ if $\hat{U}^{p}$ is differentiable at $\boldsymbol{\sigma}$.

The existence of a plastic work function $W^{p}$, which bears the same relation to $U^{p}$ as $W$ does to $U$, is not as readily deduced since the function $\boldsymbol{\sigma}=\tilde{\boldsymbol{\sigma}}(\Delta \mathbf{p})$ is multivalued. We resolve this by appealing to the concept of a conjugate or polar function (Aubin and Ekeland (1984), Ekeland and Temam (1976), Chapter 1, Sec. 5, Rockafellar (1970), Part III). Given the convex function $\hat{U}^{p}: R^{N \times N} \rightarrow R$ and a point $\sigma$, we define the conjugate function $\hat{W}^{p}: R^{N \times N} \rightarrow R$ of $\hat{U}^{p}$ by $\hat{W}^{p}(\mathbf{q})=\boldsymbol{\sigma} \cdot \mathbf{q}-\hat{U}^{p}(\boldsymbol{\sigma})$, where $\mathbf{q} \in \partial \hat{U}(\boldsymbol{\sigma})$. The following properties of $\hat{W}^{p}$ then follow from the definition of $\hat{W}^{p}$.

THEOREM 4.2. (a) If $\mathbf{q} \in \partial \hat{U}^{p}(\boldsymbol{\sigma})$ then $\boldsymbol{\sigma} \in \partial \hat{W}^{p}(\mathbf{q})$.

(b) $\hat{W}^{p}$ is convex.

Proof. (a) Let $\mathbf{q} \in \partial \hat{U}^{p}(\boldsymbol{\sigma})$ and $\mathbf{r} \in \partial \hat{U}^{p}(\tau)$. Then

$$
\begin{aligned}
\hat{W}^{p}(\mathbf{r})-\hat{W}^{p}(\mathbf{q})-\boldsymbol{\sigma} \cdot(\mathbf{r}-\mathbf{q}) & =\mathbf{r} \cdot \tau-\hat{U}^{p}(\tau)-\mathbf{q} \cdot \boldsymbol{\sigma}+\hat{U}^{p}(\boldsymbol{\sigma})-\boldsymbol{\sigma} \cdot(\mathbf{r}-\mathbf{q}) \\
& =\hat{U}^{p}(\boldsymbol{\sigma})-\hat{U}^{p}(\tau)-\mathbf{r} \cdot(\boldsymbol{\sigma}-\tau) \geqslant 0 .
\end{aligned}
$$

(b) follows in the same way as the proof of Theorem 3.1(b).

We thus have available a function $\hat{W}^{p}$, called henceforth the plastic work function, which can be defined and given desirable properties without the need to work with the multivalued function $\tilde{\boldsymbol{\sigma}}(\Delta \mathbf{p})$. There are of course values of $\Delta \mathbf{p}$ for which $\tilde{\boldsymbol{\sigma}}(\Delta \mathbf{p})$ is single-valued, and for such cases $\boldsymbol{\sigma}=\partial \hat{W}^{p} / \partial(\Delta \mathbf{p})=\partial \hat{W}^{p} / \partial \mathbf{p}$. But $\hat{W}$ is not differentiable everywhere, in particular, not at $\mathbf{p}=\mathbf{p}^{0}$, at which point $\partial \hat{W}^{p}(\mathbf{0})$ corresponds to the set of all stresses $\boldsymbol{\sigma}$ such that an elastic path exists from $\boldsymbol{\sigma}^{0}$ to $\boldsymbol{\sigma}$.

5. Determination of extremal stress paths. Extremal stress paths have previously been determined (see Ponter (1968), Martin (1971), Soechting and Lance (1969), and for a review, Martin (1975)) for elastic-plastic materials which undergo kinematic hardening and isotropic hardening, with the assumption that the stress paths emanate from $\boldsymbol{\sigma}^{0}=\mathbf{0}$. This treatment excludes, for example, the possibility of finding the extremal path from a point $\boldsymbol{\sigma}^{0}$ to a point $\boldsymbol{\sigma}$ in a kinematically hardening material for which the current yield surface does not enclose the origin. The present formulation allows us to find extremal stress paths from any point $\sigma^{0}$ to any point $\boldsymbol{\sigma}$; our methods are different but the conclusions differ very little from those given earlier. By way of an example we determine here extremal stress paths for kinematically hardening materials only.

The problem, then, is the following. Given an elastic-plastic kinematically hardening material which obeys Postulates 1 to 3 in Sec. 2, find the extremal paths between two points $\boldsymbol{\sigma}^{\sigma}$ and $\boldsymbol{\sigma}$. For such a material the yield function $\phi$ depends on $\boldsymbol{\sigma}$ and $\mathbf{p}$ in the form

$$
\phi=\phi(\boldsymbol{\sigma}, \mathbf{p})=\bar{\phi}(\boldsymbol{\sigma}-h \mathbf{p})=0,
$$


where $h$ is a positive constant. Without loss of generality, we assume that $\sigma^{0}$ lies inside the current yield surface and $\sigma$ lies outside of it (Fig. 5.1), that is,

$$
\phi\left(\boldsymbol{\sigma}^{0}, \mathbf{p}^{0}\right)<0, \quad \phi\left(\boldsymbol{\sigma}, \mathbf{p}^{0}\right)>0 .
$$

Then an extremal path will consist of two segments, one going from $\boldsymbol{\sigma}^{0}$ to a point $\boldsymbol{\sigma}^{\prime}$, say, on the yield surface $\phi\left(\boldsymbol{\sigma}, \mathbf{p}^{0}\right)=0$ and the remainder of the path going from $\boldsymbol{\sigma}^{\prime}$ to $\boldsymbol{\sigma}$. Of course any path between $\boldsymbol{\sigma}^{0}$ and $\boldsymbol{\sigma}^{\prime}$ which remains inside the yield surface will suffice since such a path will be an elastic one, and therefore extremal. The problem that remains is one of locating $\boldsymbol{\sigma}^{\prime}$, and of determining the path between $\boldsymbol{\sigma}^{\prime}$ and $\boldsymbol{\sigma}$. A formal statement of the problem is thus:

Minimize

$$
\int_{0}^{1} \Delta \mathbf{p}(t) \cdot \dot{\mathbf{f}}(t) d t
$$

subject to

$$
\phi(\boldsymbol{\sigma}, \mathbf{p})=\bar{\phi}(\boldsymbol{\sigma}-h \mathbf{p})=0,
$$

where $\mathbf{f}(t)(0 \leqslant t \leqslant 1)$ is the path between $\boldsymbol{\sigma}^{\prime}$, which is as yet unknown, and $\boldsymbol{\sigma}$. We use a Lagrange-multiplier approach and minimize

$$
J(\mathbf{p}, \mathbf{f})=\int_{0}^{1}[\Delta \mathbf{p}(t) \cdot \dot{\mathbf{f}}(t)+\lambda \phi(\mathbf{f}(t), \mathbf{p}(t))] d t,
$$

where $\lambda$ is a Lagrange multiplier. The requirement that $J$ be stationary with respect to $\mathbf{p}$ and $\mathbf{f}$ yields

$$
\begin{aligned}
0 & =\langle D J(\mathbf{p}, \mathbf{f}),(\mathbf{q}, \mathbf{g})\rangle \equiv \lim _{\theta \rightarrow 0} \theta^{-1} J(\mathbf{p}+\theta \mathbf{q}, \mathbf{f}+\theta \mathbf{g}) \\
& =\int_{0}^{1}[\Delta \mathbf{p} \cdot \mathbf{g}+\dot{\mathbf{f}} \cdot \mathbf{q}+\lambda(\mathbf{M} \cdot \mathbf{g}+\mathbf{N} \cdot \mathbf{q})] d t \\
& =\int_{0}^{1}[-\dot{\mathbf{p}} \cdot \mathbf{g}+\dot{\mathbf{f}} \cdot \mathbf{q}+\lambda(\mathbf{M} \cdot \mathbf{g}+\mathbf{N} \cdot \mathbf{q})] d t, \quad(\dot{\mathbf{p}}=\Delta \dot{\mathbf{p}}),
\end{aligned}
$$

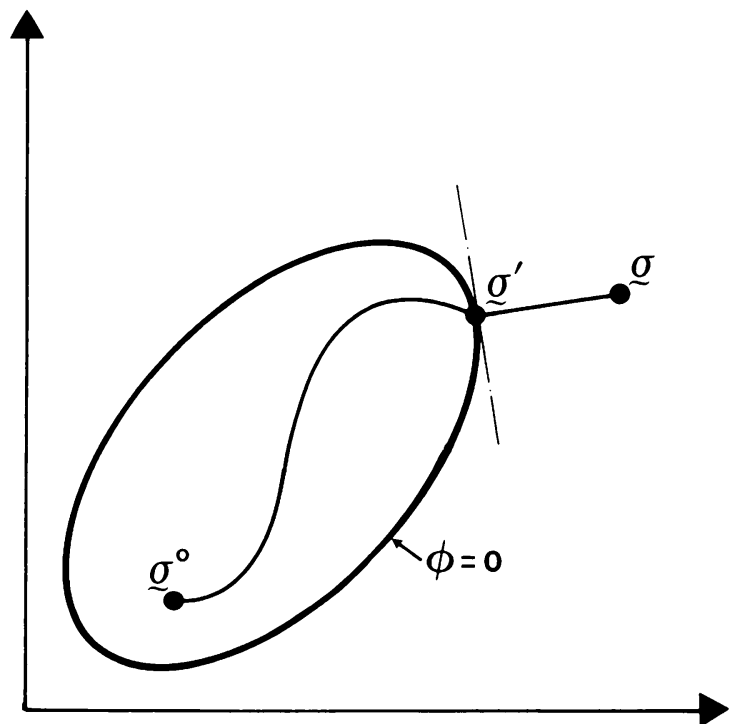

FIG. 5.1. Extremal path for a linear kinematically hardening material. 
after integrating the first term in the integrand by parts, wherein $\mathbf{M}=\partial \phi / \partial \boldsymbol{\sigma}$ and $\mathbf{N}=\partial \phi / \partial \mathbf{p}$. Since $\mathbf{g}$ and $\mathbf{q}$ are both arbitrary we must have

$$
\dot{\mathbf{p}}=\lambda \mathbf{M}, \quad \dot{\mathbf{f}}=-\lambda \mathbf{N} \text {. }
$$

But for kinematic hardening

$$
\partial \phi / \partial \boldsymbol{\sigma}=\partial \bar{\phi} / \partial \overline{\boldsymbol{\sigma}}, \quad \partial \phi / \partial \mathbf{p}=h \partial \bar{\phi} / \partial \overline{\boldsymbol{\sigma}}
$$

where $\overline{\boldsymbol{\sigma}}=\boldsymbol{\sigma}-h \mathbf{p}$, so that $\mathbf{N}=-\mathrm{h} \mathbf{M}$. Hence

$$
\dot{\mathbf{f}}=h \lambda \mathbf{M},
$$

that is, the extremal path emanates from the current yield surface at a point $\boldsymbol{\sigma}^{\prime}$ such that $\boldsymbol{\sigma}-\boldsymbol{\sigma}^{\prime}$ is codirectional with $\mathbf{M}$, the normal to $\phi$ (Fig. 5.1). Note that we also recover from (5.5) the classical normality rule.

If we make use of the von Mises yield function with kinematic hardening, then we have

$$
\phi(\boldsymbol{\sigma}, \mathbf{p})=\frac{1}{2}(\mathbf{s}-h \mathbf{p}) \cdot(\mathbf{s}-h \mathbf{p})-k^{2},
$$

where $\mathbf{s}=\boldsymbol{\sigma}-\frac{1}{3} \boldsymbol{\sigma}_{k k} \mathbf{I}$ is the deviatoric stress and $k$ is a constant. Suppose that the plastic strain associated with $\boldsymbol{\sigma}^{0}$ is $\mathbf{p}=\mathbf{p}^{0}$; then $\phi\left(\boldsymbol{\sigma}^{\prime}, \mathbf{p}^{0}\right)=0$, so that at $\left(\boldsymbol{\sigma}^{\prime}, \mathbf{p}^{0}\right), \mathbf{M}^{\prime}=$ $\partial \phi /\left.\partial \boldsymbol{\sigma}\right|_{\boldsymbol{\sigma}=\boldsymbol{\sigma}^{\prime}}=\mathbf{s}^{\prime}-h \mathbf{p}^{0}$. Now since $\dot{\mathbf{f}}=h \dot{\mathbf{p}}$ we find upon integration along the extremal path that

$$
\boldsymbol{\sigma}-\boldsymbol{\sigma}^{\prime}=h\left(\mathbf{p}-\mathbf{p}^{0}\right)
$$

so that

$$
\mathbf{p}-\mathbf{p}^{0}=\alpha\left(\mathbf{s}^{\prime}-h \mathbf{p}^{0}\right)
$$

for an appropriate constant $\alpha$. Substitution in the yield condition $\phi\left(\boldsymbol{\sigma}^{\prime}, \mathbf{p}^{0}\right)=0$ gives

$$
\frac{1}{2 \alpha^{2}}\left(\mathbf{p}-\mathbf{p}^{0}\right) \cdot\left(\mathbf{p}-\mathbf{p}^{0}\right)-k^{2}=0
$$

or

$$
\alpha=\frac{\left|\mathbf{p}-\mathbf{p}^{0}\right|}{\sqrt{2} k}
$$

so that

$$
\mathbf{s}^{\prime}(\Delta \mathbf{p})=\sqrt{2} k \frac{\Delta \mathbf{p}}{|\Delta \mathbf{p}|}+h \mathbf{p}^{0} .
$$

Finally, to obtain the complementary plastic work and plastic work functions we use

$$
\begin{aligned}
\hat{U}^{p}(\boldsymbol{\sigma}) & =\int_{0}^{1} \Delta \mathbf{p}(t) \cdot \dot{\mathbf{f}}(t) d t \\
& =h \int_{0}^{1} \Delta \mathbf{p}(t) \cdot \dot{\mathbf{p}}(t) d t=\frac{h}{2}\left(\mathbf{p} \cdot \mathbf{p}-\mathbf{p}^{0} \cdot \mathbf{p}^{0}\right)-h \mathbf{p}^{0} \cdot \Delta \mathbf{p} \\
& =\frac{h}{2} \Delta \mathbf{p} \cdot \Delta \mathbf{p} \\
& =\frac{1}{2 h}\left(\boldsymbol{\sigma}-\boldsymbol{\sigma}^{\prime}\right) \cdot\left(\boldsymbol{\sigma}-\boldsymbol{\sigma}^{\prime}\right) .
\end{aligned}
$$


Hence,

$$
\begin{aligned}
\hat{W}^{p}(\Delta \mathbf{p}) & =\boldsymbol{\sigma} \cdot \Delta \mathbf{p}-\hat{U}^{p}(\boldsymbol{\sigma}) \\
& =\frac{h}{2}|\Delta \mathbf{p}|^{2}+\boldsymbol{\sigma}^{\prime}(\Delta \mathbf{p}) \cdot \Delta \mathbf{p} \\
& =\frac{h}{2}|\Delta \mathbf{p}|^{2}+\mathbf{s}^{\prime}(\Delta \mathbf{p}) \cdot \Delta \mathbf{p}\left(\text { since } \Delta p_{k k}=0\right),
\end{aligned}
$$

where $\mathbf{s}^{\prime}(\Delta \mathbf{p})$ is given by (5.9). It is readily verified that for $\mathbf{p}-\mathbf{p}^{0} \neq \mathbf{0}, \hat{W}^{p}$ is differentiable and $\boldsymbol{\sigma}=\partial \hat{W}^{p} / \partial \Delta \mathbf{p}$. Indeed, for $\mathbf{p}-\mathbf{p}^{0} \neq \mathbf{0}$,

$$
\frac{\partial \hat{W}^{p}}{\partial \Delta \mathbf{p}}=h \Delta \mathbf{p}+\frac{\partial}{\partial \Delta \mathbf{p}}\left(\mathbf{s}^{\prime} \cdot \Delta \mathbf{p}\right)
$$

Now

$$
\begin{aligned}
\frac{\partial}{\partial \mathbf{p}}\left(\mathbf{s}^{\prime} \cdot \Delta \mathbf{p}\right)= & \sqrt{2} k\left[\frac{\Delta \mathbf{p}}{r}+\frac{\Delta \mathbf{p}}{r}-\frac{(\Delta \mathbf{p} \cdot \Delta \mathbf{p})}{r^{3}} \Delta \mathbf{p}\right]+h \mathbf{p} \\
& \left(r=\left|\mathbf{p}-\mathbf{p}^{0}\right|=|\Delta \mathbf{p}|\right) \\
= & \sqrt{2} k \Delta \mathbf{p} / r+h \mathbf{p} .
\end{aligned}
$$

$\hat{W}^{p}$ is not differentiable at $\mathbf{0}$, a fact which reflects the multivaluedness of $\boldsymbol{\sigma}(\mathbf{0})$, and we have $\boldsymbol{\sigma}(\mathbf{0}) \in \partial \hat{W}^{p}(\mathbf{0})$.

6. A holonomic material. With a full characterization of extremal paths at our disposal we are now in a position to define a constitutive law for a nonlinear elastic material, this material behaving in the same way as an elastic-plastic material if the stress paths followed are extremal.

Given a material point with stress $\sigma^{0}$ and strain $\varepsilon^{0}$ (so that the plastic strain $\mathbf{p}^{0}$ can be found from $\mathbf{p}^{0}=\boldsymbol{\varepsilon}^{0}-\mathbf{D \sigma}^{0}$ ), we define a holonomic material to be one for which the stress $\boldsymbol{\sigma}$, the $\operatorname{strain} \varepsilon$, and the plastic strain $\mathbf{p}$ are related by

$$
\begin{gathered}
\boldsymbol{\sigma}=\mathbf{C}(\boldsymbol{\varepsilon}-\mathbf{p})=\mathbf{C}(\Delta \boldsymbol{\varepsilon}-\Delta \mathbf{p})+\mathbf{C} \mathbf{e}^{0}, \\
\boldsymbol{\sigma} \in \partial \hat{W}^{p}(\Delta \mathbf{p}),
\end{gathered}
$$

where $\mathbf{C}$ is the fourth-order elasticity tensor inverse to $\mathbf{D}$ (see (2.1)). Constitutive equations dual to (6.2) may be set up using the complementary plastic work function, but in conventional displacement-based problems, (6.1) is more useful.

In the case of a holonomic law derived from a kinematically hardening elastic-plastic material we can be more explicit about (6.2); in this case (6.2) can be written alternatively as

$$
\begin{gathered}
\boldsymbol{\sigma}(\Delta \mathbf{p})=h\left(\Delta \mathbf{p}+\mathbf{p}^{0}\right)+\sqrt{2} k \Delta \mathbf{p} /|\Delta \mathbf{p}|, \quad \Delta \mathbf{p} \neq \mathbf{0}, \\
\boldsymbol{\sigma}(\mathbf{0})-h \mathbf{p}^{0} \in \partial \tilde{W}(\mathbf{0})
\end{gathered}
$$

where $\tilde{W}(\Delta \mathbf{p})=\sqrt{2} k|\Delta \mathbf{p}|$ is the nondifferentiable part of $\hat{W}^{p}$. It is a straightforward matter to show that Eqns. (6.3) and (6.4) are equivalent to the following conditions:

$$
\begin{gathered}
|\boldsymbol{\sigma}-h \mathbf{p}|<\sqrt{2} k \Rightarrow \Delta \mathbf{p}=0, \\
|\boldsymbol{\sigma}-h \mathbf{p}|=\sqrt{2} k \Rightarrow \text { there exists } \beta>0 \text { such that } \Delta \mathbf{p}=\beta(\boldsymbol{\sigma}-h \mathbf{p}) .
\end{gathered}
$$

This is illustrated for a one-dimensional situation in Fig. 6.1. 

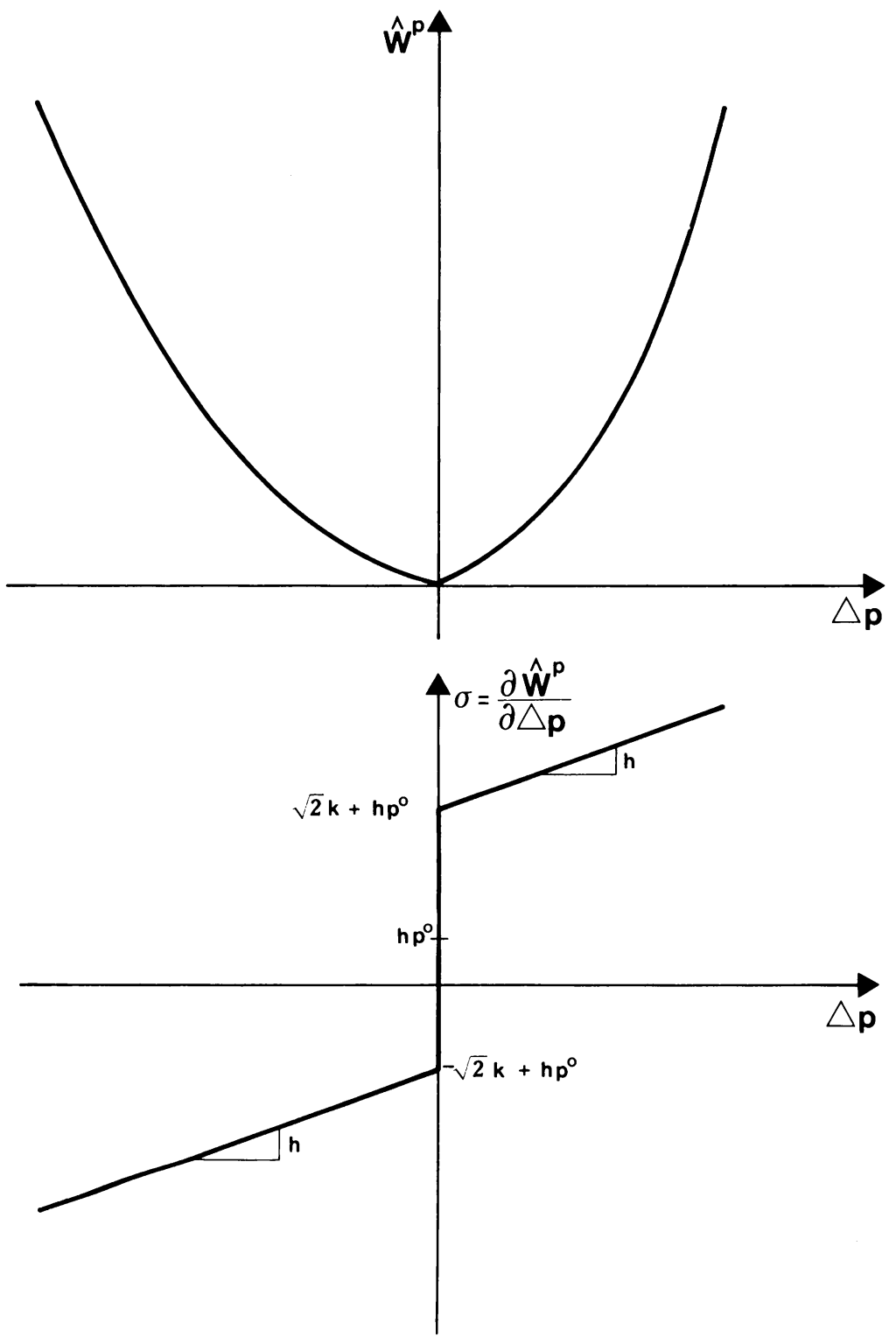

FIG. 6.1. A one-dimensional representation of the plastic work function $\hat{W}^{p}$ and its derivative, the stress.

In a companion paper (Reddy and Griffin (1986)) we examine variational formulations of boundary-value problems based on (6.1), (6.2); the appropriate variational formulation is shown to be a variational inequality of the second kind.

Convergence of finite element approximations of these boundary-value problems is also examined. Then in another contribution (Griffin, Reddy, and Martin (1986)) we undertake a detailed numerical investigation of the efficacy of using a holonomic law such as (6.1), (6.2) in an incremental fashion to determine approximations to the response of elastic-plastic bodies for arbitrary load histories. 
Acknowledgment. The authors wish to acknowledge the financial assistance of the Foundation for Research and Development of the CSIR.

\section{REFERENCES}

[1] Aubin, J.-P. and Ekeland, I., Applied Nonlinear Analysis, Wiley, New York, 1984

[2] Budiansky, B., A reassessment of deformation theories of plasticity, J. Appl. Mech. 26, 259 (1959)

[3] Cohn, M. Z. and Maier, G. (eds.), Engineering Plasticity by Mathematical Programming (Proc. Nato Adv. Studies Inst., Univ. of Waterloo, Aug. 1977), Pergamon, New York, 1979

[4] Ekeland, I. and Temam, R. Convex Analysis and Variational Problems, North-Holland, Amsterdam, 1976

[5] Griffin, T. B., Reddy, B. D., and Martin, J. B., A numerical study of holonomic approximations to problems in plasticity, Internat. J. Numer. Methods Engrg. (to appear)

[6] Hencky, H., Zur Theorie plastischer Deformationen und der hierdurch in Material herforgerufenen Nachspannungen, Zeit. Ang. Math. Mech. 4, 323 (1924)

[7] Kachanov, L. M., Fundamentals of the Theory of Plasticity, Mir, Moscow, 1974

[8] Maier, G., Complementary plastic work theorems in piecewise-linear elastoplasticity, Internat. J. Solids and Structures 5, 261 (1969)

[9] Martin, J. B., A complementary energy bounding theorem for time-independent materials. In Developments in Theoretical and Applied Mechanics (ed. D. Frederick and E. H. Harris). Pergamon, New York, 1971, p. 517

[10] Martin, J. B., Plasticity: Fundamentals and General Results, MIT Press, Cambridge, Mass., 1975

[11] Nadai, A. L., Plasticity, McGraw-Hill, New York, 1931

[12] Ponter, A. R. S., Convexity conditions and energy theorems for time-independent materials, J. Mech. Phys. Solids 16, 283 (1968)

[13] Ponter, A. R. S. and Martin, J. B., Some extremal properties and energy theorems for inelastic materials and their relationship to the deformation theory of plasticity, J. Mech. Phys. Solids 20, 281 (1972)

[14] Rockafellar, R. T., Convex Analysis, Princeton Univ. Press, Princeton, 1970

[15] Reddy, B. D. and Griffin, T. B., Variational principles and convergence of finite element approximations of a holonomic elastic-plastic problem (submitted for publication)

[16] Soechting, J. F. and Lance, R. H., A bounding principle in the theory of work-hardening plasticity, J. Appl. Mech. 36, 228 (1969) 